\title{
BMJ Open Exploring the facilitators and barriers to using an online infertility risk prediction tool (FoRECAsT) for young women with breast cancer: a qualitative study protocol
}

\author{
Zobaida Edib (1) ,1,2 Yasmin Jayasinghe, ${ }^{1,2,3}$ Martha Hickey, ${ }^{1,2}$ Lesley Stafford,,${ }^{4,5}$ \\ Richard A Anderson, ${ }^{6} \mathrm{H}$. Irene Su, ${ }^{7}$ Kate Stern, ${ }^{8,9}$ Christobel Saunders, ${ }^{10}$ \\ Antoinette Anazodo, ${ }^{11,12}$ Mary Macheras-Magias, ${ }^{13}$ Shanton Chang, ${ }^{14}$ \\ Patrick Pang ${ }^{14}$ Franca Agresta,,${ }^{8,9}$ Laura Chin-Lenn, ${ }^{15}$ Wanyuan Cui, ${ }^{16}$ \\ Sarah Pratt, ${ }^{17}$ Alex Gorelik, ${ }^{18,19}$ Michelle Peate ${ }^{1,2}$
}

To cite: Edib Z, Jayasinghe $Y$, Hickey $\mathrm{M}$, et al. Exploring the facilitators and barriers to using an online infertility risk prediction tool (FoRECAsT) for young women with breast cancer: a qualitative study protocol. BMJ Open 2020;10:e033669. doi:10.1136/ bmjopen-2019-033669

- Prepublication history for this paper is available online. To view these files, please visit the journal online (http://dx.doi org/10.1136/bmjopen-2019033669).

Received 16 August 2019 Revised 09 January 2020 Accepted 13 January 2020

Check for updates

(C) Author(s) (or their employer(s)) 2020. Re-use permitted under CC BY-NC. No commercial re-use. See rights and permissions. Published by BMJ.

For numbered affiliations see end of article.

Correspondence to

Dr Zobaida Edib;

zedib@student.unimelb.edu.au

\section{ABSTRACT}

Introduction As cancer treatments may impact on fertility, a high priority for young patients with breast cancer is access to evidence-based, personalised information for them and their healthcare providers to guide treatment and fertility-related decisions prior to cancer treatment. Current tools to predict fertility outcomes after breast cancer treatments are imprecise and do not offer individualised prediction. To address the gap, we are developing a novel personalised infertility risk prediction tool (FoRECAsT) for premenopausal patients with breast cancer that considers current reproductive status, planned chemotherapy and adjuvant endocrine therapy to determine likely posttreatment infertility. The aim of this study is to explore the feasibility of implementing this FoRECAsT tool into clinical practice by exploring the barriers and facilitators of its use among patients and healthcare providers.

Methods and analysis A cross-sectional exploratory study is being conducted using semistructured in-depth telephone interviews with 15-20 participants each from the following groups: (1) premenopausal patients with breast cancer younger than 40, diagnosed within last 5 years, (2) breast surgeons, (3) breast medical oncologists, (4) breast care nurses (5) fertility specialists and (6) fertility preservation nurses. Patients with breast cancer are being recruited from the joint Breast Service of three affiliated institutions of Victorian Comprehensive Cancer Centre in Melbourne, Australia-Peter MacCallum Cancer Centre, Royal Melbourne Hospital and Royal Women's Hospital, and clinicians are being recruited from across Australia. Interviews are being audio recorded, transcribed verbatim and imported into qualitative data analysis software to facilitate data management and analyses.

Ethics and dissemination The study protocol has been approved by Melbourne Health Human Research Ethics Committee, Australia (HREC number: 2017.163). Confidentiality and privacy are maintained at every stage of the study. Findings will be disseminated through peerreviewed scholarly and scientific journals, national and international conference presentations, social media,
Strengths and limitations of this study

Obtaining representative stakeholder feedback is an essential step in ensuring that a risk prediction tool is feasible and acceptable for use in clinical practice.

- This tool could be adapted to newer breast cancer treatments and for other cancers.

- Non-probability sampling may increase the risk of selection bias.

- Recruitment is limited to patients with breast cancer where fertility was discussed prior to cancer treatment, findings may not be applicable where fertility was not discussed.

- This study is being conducted in the Australian setting, findings may not be generalisable to different health settings.

broadcast media, print media, internet and various community/stakeholder engagement activities.

\section{INTRODUCTION}

Globally, breast cancer is the most frequent cancer diagnosis in reproductive-aged women, with approximately 100000 women younger than 40 years diagnosed annually worldwide, representing one-quarter of new breast cancer cases. ${ }^{1-3}$ In Australia, most women are diagnosed with early-stage disease, and with current treatment, the 5 -year survival rate for women diagnosed with breast cancer is often excellent $(90.8 \%) .{ }^{4}$ Recommended treatment can include gonadotoxic chemotherapeutic agents and thus poses a potential threat to fertility by destroying the eggs stored in the ovaries. ${ }^{5} 6$ If the number of eggs is substantially depleted, early menopause and/ or permanent infertility can result, ${ }^{7}$ and will 
commonly present as amenorrhoea (ie, cessation of the menstrual cycle) ${ }^{8}$ Infertility and/or early menopause is a recognised long-term adverse effect of breast cancer treatment in premenopausal women and has serious implications for the survivorship experience of these women. ${ }^{89}$

Fertility is well established to be a priority for many young premenopausal patients with breast cancer. More than half are concerned about their future fertility, and $50 \%-76 \%$ wish to consider pregnancy following cancer treatment. ${ }^{10-12}$ This number is likely to increase with the social trends of delayed motherhood until older reproductive ages. ${ }^{13} 14$ Concerns about the potential risk of infertility and the inability to conceive in the future have direct implications for treatment efficacy and long-term physical and emotional health ${ }^{10}{ }^{15-19}$ - specifically it may influence patients to choose less optimal adjuvant therapies to reduce impact on fertility ${ }^{10} 112021$ or the uptake of fertility preservation options despite potential physical, emotional and financial burden. ${ }^{22-24}$ Young women with breast cancer actively seek and desire knowledge, and improved information translates into better health outcomes. ${ }^{25}{ }^{26}$ Core to making informed fertility-related decisions is an understanding of the risk of infertility, but the currently available information about fertility outcomes following breast cancer treatment can only determine broad risk categories (eg, intermediate risk: $30 \%-70 \%$ risk of infertility ${ }^{27}$ and individual factors which are known to affect fertility in women (eg, age, body mass index, smoking, previous fertility, serum ovarian markers) are not included in the risk prediction. There is a gap in personalised information to inform young patients with breast cancer about likely fertility outcomes after treatment. ${ }^{28-30}$ To meet their unmet information needs, young patients frequently use the internet to seek more accessible and consolidated information about post-treatment reproductive consequences. ${ }^{31}$ Therefore, an evidencebased and individualised online risk prediction tool may provide reliable and easy-to-access information to address the gap and better manage the fertility-related needs. ${ }^{32} 33$

Accurate prediction of infertility after breast cancer treatment is complex and requires consideration of baseline fertility and the likely impact of planned cancer treatments on fertility. ${ }^{28}$ There is growing evidence that baseline fertility indicators prior to breast cancer treatment may predict the likelihood of developing amenorrhoea after treatment. ${ }^{29} 3435$ However, no previous studies have included baseline demographic and lifestyle factors, as well as serum ovarian markers and cancer treatment factors, all together, to predict fertility. To address this gap, we are developing the fertility after cancer predictor (FoRECAsT) tool for young patients with breast cancer which considers both baseline fertility indicators and the impact of planned cancer treatment on fertility. Based on the input information, it will provide an individualised risk of amenorrhoea at different time points after initial treatment (12 months, 24 months, 36 months, 48 and 60 months) to assess longitudinal changes in infertility risk, with amenorrhoea being a surrogate marker for infertility. The tool will allow users to input individual data (baseline demographic and lifestyle factors, serum ovarian markers and recommended breast cancer treatment) to determine a personalised risk of infertility after breast cancer treatment.

There are two key parts to the FoRECAsT toolthe algorithm development and the user interface. To develop the risk prediction algorithm (part one), authors from studies exploring variables related to fertility at baseline and impact of breast cancer treatment (table 1) ) $^{296-44}$ have been invited to join the FoRECAsT Collaboration and contribute their data to the FoRECAsT database and these data are being used to build a predictive model.

The algorithm will use Bayesian inference technique, which is the preferred method in complex algorithm development, in combination with Markov chain Monte Carlo simulations. ${ }^{45-49}$ From the algorithm, a working prototype of the tool will be developed (part two) as a proof of concept. To achieve part 2 and ensure that the tool is widely used clinically to facilitate oncofertility decision making, the user interface will be developed in consultation with stakeholders including patients and patient advocacy groups. This protocol reports on a key aspect of this consultation process. Findings from this part of the study will be used to design the user interface of the FoRECAsT (prototype) tool ensuring it is easy to use and understand. There are successive steps to validate the predictive algorithm and evaluate the tool prior to implementation in clinical practice.

Table 1 Candidate predictors for fertility

\begin{tabular}{ll}
\hline Lifestyle factors & Age, race, body mass index, diet, exercise, smoking, alcohol, caffeine, drugs. \\
Medical history & $\begin{array}{l}\text { Prior (in)fertility and IVF, menstruation history, tubal and gynaecological disease, endometriosis, } \\
\text { polycystic ovary syndrome, sexually transmitted infections, pelvic surgery, family history of (in)fertility and } \\
\text { menopause. }\end{array}$
\end{tabular}

Serum markers of Follicle stimulating hormone, luteinising hormone, estradiol, inhibin B, antimullerian hormone, antral follicle ovarian function count, ovarian volume.

Cancer factors Age at diagnosis, stage, receptor status, type of treatment (dose and duration).

IVF, in vitro fertilization. 


\section{Objectives}

The main purpose of this study is to explore perceptions, ideas and opinions from young patients with breast cancer and clinicians regarding the design and feasibility of implementing the FoRECAsT tool including barriers and facilitators. Findings will also inform breast cancer patients' and clinicians' preferences of where and when the FoRECAsT tool might be used.

\section{METHODS AND ANALYSIS \\ Study design}

A cross-sectional exploratory study is being conducted through semistructured in-depth telephone interviews with key stakeholders.

\section{Study participants/stakeholders}

The following stakeholders are included in our study:

a. Patient group: 15-20 patients with breast cancer.

b. Clinician group:

- 15-20 breast surgeons.

- 15-20 breast medical oncologists.

- 15-20 breast care nurses.

- 15-20 fertility specialists.

- 15-20 fertility preservation nurses.

The sample size is an appropriate minimum sample required for meaningful outcomes. However, as per qualitative methodology, participants will continue to be recruited until informational redundancy is achieved. ${ }^{50}$

\section{Eligibility criteria}

\section{Patients with breast cancer}

\section{Inclusion criteria}

To be eligible to participate patients with breast cancer must be (1) female, (2) diagnosed within the last 5 years.

(3) aged 18-40 years (4) premenopausal at breast cancer diagnosis (5) have evidence of prior discussion with a healthcare provider about the risk of developing infertility after breast cancer treatment either through referral to a fertility specialist or documented discussion inpatient notes (so as not to cause distress in those who had not had a prior discussion about potential infertility), (6) concerned about future fertility after chemotherapy and/or have not completed their family (as identified by the treatment team), (7) able to give informed written consent and (8) able to speak and understand English.

\section{Exclusion criteria}

Women with metastatic breast cancer and women diagnosed with gestational breast cancer.

\section{Clinicians}

\section{Inclusion criteria}

To be eligible to participate clinicians who: (1) have a valid Australian License for practice, (2) have at least 1 year of clinical experience in their respective discipline, (3) consult to women with breast cancer, (4) will be able to give informed written consent and (5) will able to speak and understand English.

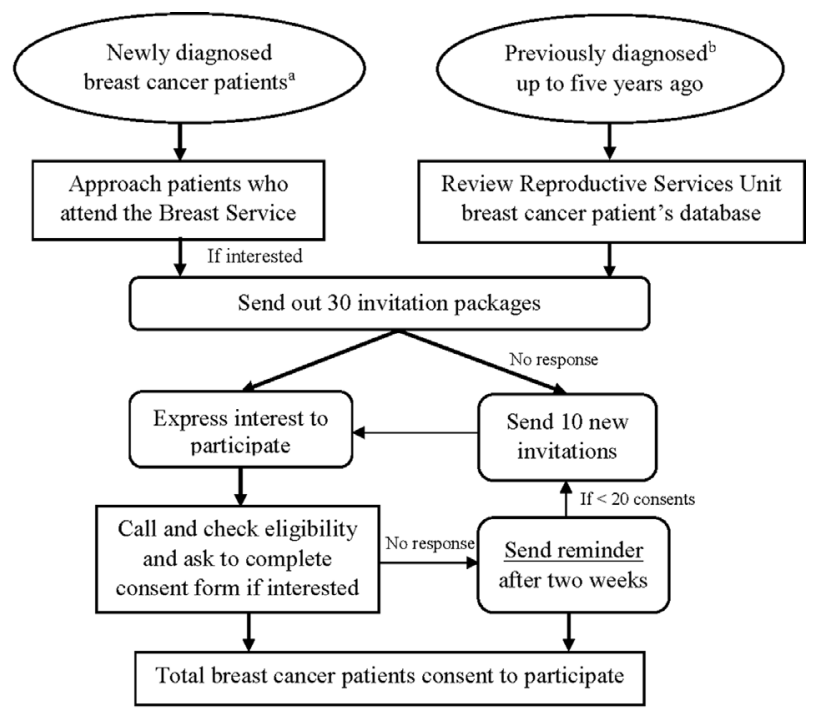

a Newly diagnosed patients are those who haven't started their chemotherapy yet 'Previously diagnosed are those who have completed the chemotherapy and diagnosed within the last five years Figure 1 Illustration of the recruitment of patients with breast cancer.

\section{Recruitment}

Recruitment started in September 2018 and is still ongoing. As per qualitative methodology, participants will continue to be recruited until informational redundancy is achieved. Patients with breast cancer are being recruited using purposive sampling by the breast care nurses from the joint Breast Service of Peter MacCallum Cancer Centre, Royal Melbourne Hospital and Royal Women's Hospital. Figure 1 illustrates the recruitment of patients with breast cancer. Clinicians are being recruited using an e-flyer through their respective online communities across Australia (except northern territory and Tasmania due to ethics committee coverage), that is, Breast Surgeons of Australia and New Zealand, Medical Oncology Group of Australia, Fertility Society of Australia, Cancer Nurses Society of Australia and McGrath Foundation. Figure 2 shows the recruitment of clinicians. Participation is voluntary, and participants may choose not to

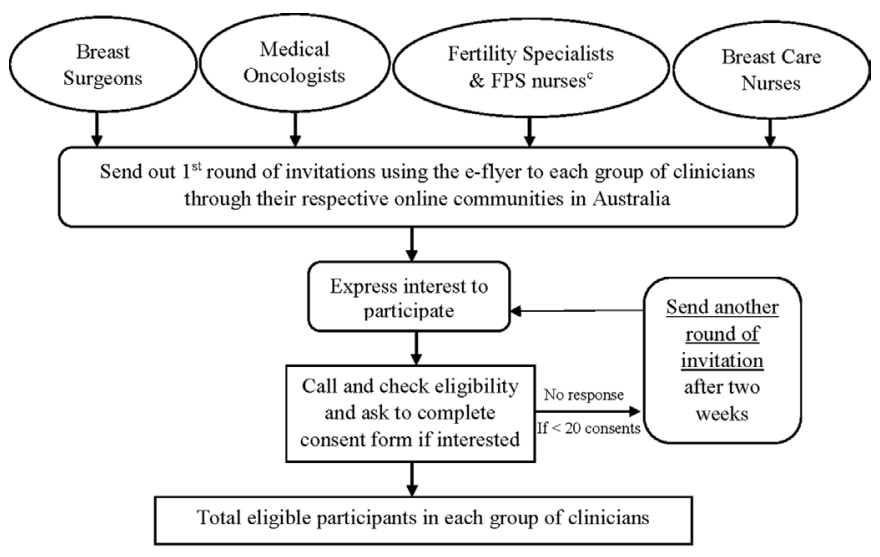

${ }^{\circ}$ Fertility preservation nurses

Figure 2 Illustration of the recruitment of clinicians. 
participate in the study or may withdraw from the study at any time. There will be an opportunity for participants to ask the research team any questions regarding the study. Invited participants, who do not respond, will be followed up with a second invitation 2 weeks after initial contact.

\section{Data collection}

In-depth telephone interviews are guided by semistructured interview schedules and carried out by the research team. Consented participants are asked to review the draft FoRECAsT tool to provide their feedback. The interview schedules are structured in consultation with clinical experts and qualitative research specialists based on Aizen's theory of planned behaviour. ${ }^{51}$ They are customised to the level of stakeholders to allow questioning strategy and conversations to be more flexible.

Each interview is anticipated to last for 15-20 min. Interviews are audio recorded on a portable, electronic digital voice recorder (Olympus VN-731PC) and transcribed verbatim. The audio recordings and transcripts have been securely stored in a password-protected folder on The University of Melbourne server with access permitted to authorised personnel only. Verbal informed consents are obtained for audio recording the interview. Interviews will be conducted until saturation is reached.$^{50}$ Patients and clinicians who consent to be interviewed have been offered the opportunity to view a copy of the transcripts prior to data analysis.

\section{Patient and public involvement}

The study is supported by a consumer/patient who is a part of the working party and involved in the design of the study, and preparation of all the study materials from the patient's perspective. All interested participants will be sent a summary report of the results via email or mail with deidentified aggregated findings.

\section{Outcome measures}

Sociodemographic data are collected from each participating patient with breast cancer and clinician. Patients with breast cancer are asked about their current age, the highest level of education attained, employment status, stage of cancer, relationship status and fertility history. Clinicians are asked about their age, years of clinical experience and proportion of patients seen with breast cancer.

Qualitative data are focusing on five topics (table 2):

1. Interest in using the tool.

2. Access and confidentiality.

3. User attributes.

4. The potential impact of the tool on consultation.

5. Anticipated outcomes and benefits.

\section{Data analysis}

The processes of data collection and data analysis are ongoing. Transcripts are being imported into a qualitative data analysis software (QRS NVivo V.12-QRS International, Doncaster, Victoria, Australia) to facilitate data management and analyses. The five broad areas are developed based on the theoretical framework of planned behaviour. ${ }^{51}$ Transcripts are coded line-by-line identifying keywords, concepts and reflections in accordance with the framework of Miles and Huberman, ${ }^{52}$ a widely used framework for qualitative research methodology. Coding is being conducted using an iterative process: starting with coding for broad themes, before coding into hierarchical categories and subthemes.

To ensure the integrity and consistency of the codes and reduce bias, codes will be reviewed by the qualitative research specialist. The research team will discuss the coding tree and reach consensus. Subsequently, content analysis will also be performed for each code, to support results from thematic analyses by identifying essential aspects of the content and highlighting the recurrence of themes, to present results clearly and effectively. A final list of themes and subthemes will be determined through patterns as soon as further data that will emerge from the study add little to the emerging theory. Theoretical saturation is reached once no new themes emerge. Results will be reported according to the consolidated criteria for reporting qualitative research developed by Tong et $a l^{53}$

Table 2 Semistructured interviews topic guides for participants

\section{Broad topics Specific topics}

1. Interest in using the infertility risk prediction tool

Extent of information received/delivered about risk of infertility, decision making with *current infertility risk calculator', perceived satisfaction in using current calculators, interest in having a more accurate infertility risk prediction tool

2. Access and confidentiality

Requirements around access and user interface, security, confidentiality of input information, technical skill.

$\begin{array}{ll}\text { 3. User attributes } & \text { Perceptions of ease of use and preferences for data entry. } \\ \begin{array}{ll}\text { 4. Impact on fertility consultation } & \text { Perceptions of impact on fertility consultation. } \\ \text { 5. Anticipated outcomes and benefits } & \begin{array}{l}\text { Benefits of using a more accurate tool, barriers and additional suggestions to better } \\ \text { meet fertility-related needs. }\end{array}\end{array}\end{array}$

${ }^{*}$ Current infertility risk calculator' refers to the commonly used existing calculator for fertility risk prediction following breast cancer treatment. $^{27}$ 


\section{ETHICS AND DISSEMINATION}

This study will be conducted in compliance with the National Health and Medical Research Council National Statement on Ethical Conduct in Human Research, the Australian Code for the Responsible Conduct of Research and the Declaration of Helsinki.

\section{Confidentiality}

Confidentiality and privacy are maintained at every stage of the study. Individual participants will not be identifiable to any other members of their group or anyone else in the wider community. Participants are approached, recruited and contacted in a confidential, one-to-one manner and no public dissemination of participants' details will occur. Contact details for the researchers and relevant ethics committee(s) are provided to address any questions or concerns participants may have. Audio recordings and individual transcripts are being stored on a password protected and secured The University of Melbourne server, which is backed up daily. Study-related records will be retained in a secure storage facility for at least 7 years after the completion of the research as required by the Australian National Health and Medical Research Council.

\section{Dissemination}

Only deidentified results will be published. The results will be actively disseminated through peer-reviewed scholarly and scientific journals, national and international conference presentations, social media, broadcast media, print media, internet and various community/stakeholder engagement activities. The consumer/patient will also provide comment on the findings and contribute to the dissemination plan via consumer websites such as Breast Cancer Network Australia.

\section{STRENGTHS AND LIMITATIONS OF THIS STUDY}

This will be the first personalised tool considering baseline demographic and lifestyle factors, serum ovarian markers and cancer treatment factors all together in predicting the impact of breast cancer treatments on fertility. Strengths of this study include codesign the tool with patients' and healthcare professionals' needs and preferences in mind. This tool could potentially be implemented globally with adaptation to newer breast cancer treatment. Additionally, the tool could be adapted for other cancer treatments.

Limitations include the use of non-probability sampling to recruit patients with breast cancer, which may increase selection bias. ${ }^{54}$ Recruitment is limited to patients with breast cancer where fertility was discussed prior to cancer treatment and our findings may not be applicable to circumstances where fertility was not discussed. Also, our findings cannot be generalised to patients with breast cancer from more diverse cultural and linguistic backgrounds and those with advanced breast cancer.
Author affiliations

${ }^{1}$ Obstetrics \& Gynaecology, The University of Melbourne, Melbourne, Victoria, Australia

${ }^{2}$ Obstetrics \& Gynaecology, The Royal Women's Hospital, Parkville, Victoria, Australia ${ }^{3}$ Department of Paediatric and Adolescent Gynaecology, The Royal Children's Hospital, Parkville, Victoria, Australia

${ }^{4}$ Melbourne School of Psychological Sciences, The University of Melbourne, Melbourne, Victoria, Australia

${ }^{5}$ Centre for Women's Mental Health, The Royal Women's Hospital, Parkville, Victoria, Australia

${ }^{6}$ MRC Centre for Reproductive Health, The University of Edinburgh, Edinburgh, UK ${ }^{7}$ Department of Obstetrics, Gynecology and Reproductive Sciences, University of California San Diego, San Diego, California, USA

${ }^{8}$ Melbourne IVF, East Melbourne, Victoria, Australia

${ }^{9}$ Reproductive Services, The Royal Women's Hospital, Parkville, Victoria, Australia

${ }^{10}$ School of Surgery, The University of Western Australia, Perth, Western Australia, Australia

${ }^{11}$ Sydney Children's Hospital, School of Women's and Children's Health, UNSW, Sydney, New South Wales, Australia

${ }^{12}$ Nelune Comprehensive Cancer Centre, Prince of Wales Children's Hospital, Randwick, New South Wales, Australia

${ }^{13}$ Breast Cancer Network Australia, Camberwell, Victoria, Australia

${ }^{14}$ School of Computing and Information Systems, The University of Melbourne, Melbourne, Victoria, Australia

${ }^{15}$ Department of General Surgery, The Royal Melbourne Hospital, Melbourne, Victoria, Australia

${ }^{16}$ Department of Medical Oncology, Peter MacCallum Cancer Centre, Melbourne, Victoria, Australia

${ }^{17}$ Breast Service, Peter MacCallum Cancer Centre, Melbourne, Victoria, Australia

${ }^{18}$ Department of Medicine, The Royal Melbourne Hospital, The University of Melbourne, Melbourne, Victoria, Australia

${ }^{19}$ School of Behavioural and Health Sciences, Australian Catholic University, Melbourne, Victoria, Australia

\section{Twitter Patrick Pang @docpang}

Acknowledgements MP and YJ both are currently supported by an MDHS Fellowship, The University of Melbourne. At the time of this study, MP was supported by a National Breast Cancer Foundation Early Career Fellowship (ECF15-005). MH is supported by a National Health and Medical Research Council of Australia (NHMRC) Practitioner Fellowship and is part of the Women Choosing Surgical Prevention (WISP) investigator team funded by Stand Up to Cancer. The authors would like to thank FoRECAsT Consortium for contributing their datasets to develop the infertility risk prediction algorithm.

Collaborators FoRECAsT Consortium: Dr Michelle Peate, Dr Zobaida Edib, Prof Martha Hickey, Dr Yasmin Jayasinghe, Dr Shanton Chang, Dr Patrick Pang, Prof John Hopper, Dr Kevin Nguyen, Dr. Gillian Dite, Prof Uwe Aickelin, Xuetong Wu, Dr Hadi Akbarzadeh Khorshidi, Prof Ann Partridge, Dr Fabien Reyal, Ms Franca Agresta, Dr Genia Rozen, A/Prof Kate Stern, Dr Wanda Cui, Prof Kelly-Anne Phillips, Dr Laura Chin-Lenn, Dr Lesley Stafford, Dr Anne-Sophie Hamy, Dr Antoinette Anazodo, A/ Prof H. Irene Su, Prof Richard Anderson, Prof Scott Nelson, Ms Alexandra Gorelik, Prof Liz Sullivan, Dr Alessandro Minisini, Prof Fabio Puglisi, Dr. Claudia Bozza, Prof Christobel Saunders, Dr Kathryn J. Ruddy, Dr Fergus J. Couch, Dr Janet E. Olson, Dr Isabelle Demeestere, Dr Margherita Condorelli, Dr Matteo Lambertini, Dr Michail Ignatiadis,Dr Barbara Pistilli, Ms Mary Macheras-Magias.

Contributors MP conceived the research idea, participated in the design of the study, development of all study documents, ethical approval process and reviewed this manuscript. ZE participated in the design of the study, development of all study documents, ethical approval process, study coordination and drafted this manuscript. YJ participated in the design of the study, development of all study documents and reviewed this manuscript. MH, LS, RAA, HIS, KS, CS, AA, MM-M, SC, PP and FA participated in the review of all study documents and the manuscript. LC- $L$ and SP participated in ethics approval process and reviewed the manuscript. WC and AG reviewed the manuscript. All authors read and approved the final manuscript.

Funding This Research Project/Program was supported by the Victorian Government through a Victorian Cancer Agency (Early Career Seed Grant) awarded to Dr Peate.

Competing interests None declared. 
Patient consent for publication Not required.

Ethics approval The study protocol has been reviewed and approved by the Human Research Ethics Committee of the Melbourne Health, Australia (HREC number: 2017.163).

Provenance and peer review Not commissioned; externally peer reviewed.

Open access This is an open access article distributed in accordance with the Creative Commons Attribution Non Commercial (CC BY-NC 4.0) license, which permits others to distribute, remix, adapt, build upon this work non-commercially, and license their derivative works on different terms, provided the original work is properly cited, appropriate credit is given, any changes made indicated, and the use is non-commercial. See: http://creativecommons.org/licenses/by-nc/4.0/.

ORCID iD

Zobaida Edib http://orcid.org/0000-0001-7381-7351

\section{REFERENCES}

1 Franasiak JM, Scott RT. Demographics of cancer in the reproductive age female. cancer and fertility. Springer, 2016: 11-19.

2 Ferlay J, Soerjomataram I, Dikshit R, et al. Cancer incidence and mortality worldwide: sources, methods and major patterns in GLOBOCAN 2012. Int J Cancer 2015;136:E359-86.

3 Australian Institute of Health and Welfare. Cancer in Australia 2017. Cancer series no.101. Cat. no. CAN 100. Canberra: AlHW, 2017.

4 Australian Institute of Health and Welfare. Cancer in Australia 2019. Cancer series no.119. Cat. no. CAN 123. Canberra: AlHW, 2019.

5 Codacci-Pisanelli G, Del Pup L, Del Grande M, et al. Mechanisms of chemotherapy-induced ovarian damage in breast cancer patients. Crit Rev Oncol Hematol 2017;113:90-6.

6 Nguyen Q-N, Zerafa N, Liew SH, et al. Loss of PUMA protects the ovarian reserve during DNA-damaging chemotherapy and preserves fertility. Cell Death Dis 2018;9:618.

7 Findlay JK, Hutt KJ, Hickey M, et al. What is the "ovarian reserve"? Fertil Steril 2015;103:628-30.

8 Jayasinghe YL, Wallace WHB, Anderson RA. Ovarian function, fertility and reproductive lifespan in cancer patients. Expert Rev Endocrinol Metab 2018;13:125-36.

9 Anderson RA, Brewster DH, Wood R, et al. The impact of cancer on subsequent chance of pregnancy: a population-based analysis. Hum Reprod 2018;33:1281-90.

10 Ruddy KJ, Gelber SI, Tamimi RM, et al. Prospective study of fertility concerns and preservation strategies in young women with breast cancer. J Clin Oncol 2014;32:1151-6.

11 Lambertini M, Goldrat O, Clatot F, et al. Controversies about fertility and pregnancy issues in young breast cancer patients. Curr Opin Oncol 2017;29:243-52.

12 Taylan E, Oktay KH. Current state and controversies in fertility preservation in women with breast cancer. World J Clin Oncol 2017;8:241

13 Cooke A, Mills TA, Lavender T. 'Informed and uninformed decision making'-Women's reasoning, experiences and perceptions with regard to advanced maternal age and delayed childbearing: A metasynthesis. Int J Nurs Stud 2010;47:1317-29.

14 Peate M, Meiser B, Hickey M, et al. The fertility-related concerns, needs and preferences of younger women with breast cancer: a systematic review. Breast Cancer Res Treat 2009;116:215-23.

15 Avis NE, Crawford S, Manuel J. Psychosocial problems among younger women with breast cancer. Psychooncology 2004;13:295-308.

16 Partridge AH, Gelber S, Peppercorn J, et al. Web-based survey of fertility issues in young women with breast cancer. J Clin Oncol 2004;22:4174-83.

17 Peate M, Meiser B, Friedlander M, et al. It's now or never: fertilityrelated knowledge, decision-making preferences, and treatment intentions in young women with breast cancer--an Australian fertility decision aid collaborative group study. J Clin Oncol 2011;29:1670-7.

18 Peate M, Meiser B, Cheah BC, et al. Making hard choices easier: a prospective, multicentre study to assess the efficacy of a fertilityrelated decision aid in young women with early-stage breast cancer. Br J Cancer 2012;106:1053-61.

19 Lambertini M, Pinto AC, Del Mastro L. Fertility issues in young breast cancer patients: what women want. J Thorac Dis 2014;6:584.

20 Llarena NC, Estevez SL, Tucker SL, et al. Impact of fertility concerns on tamoxifen initiation and persistence. J Natl Cancer Inst 2015;107:djv202.

21 Pala Sehmus, Atilgan R, Ozkan ZS, et al. Effect of varying doses of tamoxifen on ovarian histopathology, serum VEGF, and endothelin 1 levels in ovarian hyperstimulation syndrome: an experimental study. Drug Des Devel Ther 2015;9:1761.

22 Barcroft J, Dayoub N, Thong KJ. Fifteen year follow-up of embryos cryopreserved in cancer patients for fertility preservation. J Assist Reprod Genet 2013;30:1407-13.

23 Dondorp W, de Wert G, Pennings G, et al. Oocyte cryopreservation for age-related fertility loss. Hum Reprod 2012;27:1231-7.

$24 \mathrm{Li} \mathrm{N}$, Jayasinghe $\mathrm{Y}$, Kemertzis MA, et al. Fertility preservation in pediatric and adolescent oncology patients: the decision-making process of parents. J Adolesc Young Adult Oncol 2017;6:213-22.

25 Duffy CM, Allen SM, Clark MA. Discussions regarding reproductive health for young women with breast cancer undergoing chemotherapy. J Clin Oncol 2005;23:766-73.

26 Logan S, Perz J, Ussher JM, et al. A systematic review of patient oncofertility support needs in reproductive cancer patients aged 14 to 45 years of age. Psychooncology 2018;27:401-9.

27 LIVESTRONG fertility risk tool, 2017. Available: https://www. livestrong.org/we-can-help/livestrong-fertility

28 Peate M, Stafford L, Hickey M. Fertility after breast cancer and strategies to help women achieve pregnancy. Cancer Forum 2017;41.

29 Anderson RA, Rosendahl M, Kelsey TW, et al. Pretreatment anti-Müllerian hormone predicts for loss of ovarian function after chemotherapy for early breast cancer. Eur J Cancer 2013;49:3404-11.

30 Anderson RA, Mansi J, Coleman RE, et al. The utility of anti-Müllerian hormone in the diagnosis and prediction of loss of ovarian function following chemotherapy for early breast cancer. Eur J Cancer 2017;87:58-64

31 Lee K, Hoti K, Hughes JD, et al. Dr Google and the consumer: a qualitative study exploring the navigational needs and online health information-seeking behaviors of consumers with chronic health conditions. J Med Internet Res 2014;16:e262.

32 Pang PC-I, Chang S, Verspoor K, et al. Designing Health Websites Based on Users' Web-Based Information-Seeking Behaviors: A Mixed-Method Observational Study. J Med Internet Res 2016;18:e145.

33 Benedict C, Thom B, N. Friedman D, et al. Young adult female cancer survivors' unmet information needs and reproductive concerns contribute to decisional conflict regarding posttreatment fertility preservation. Cancer 2016;122:2101-9.

34 D'Avila Ângela Marcon, Biolchi V, Capp E, et al. Age, antiMüllerian hormone, antral follicles count to predict amenorrhea or oligomenorrhea after chemotherapy with cyclophosphamide. $J$ Ovarian Res 2015;8:82.

$35 \mathrm{Su} \mathrm{H}-\mathrm{Cl}$, Haunschild C, Chung K, et al. Prechemotherapy antimullerian hormone, age, and body size predict timing of return of ovarian function in young breast cancer patients. Cancer 2014;120:3691-8.

36 Liem GS, Mo FKF, Pang E, et al. Chemotherapy-Related amenorrhea and menopause in young Chinese breast cancer patients: analysis on incidence, risk factors and serum hormone profiles. PLoS One 2015;10:e0140842.

37 Anderson RA, Cameron DA. Pretreatment serum anti-Müllerian hormone predicts long-term ovarian function and bone mass after chemotherapy for early breast cancer. J Clin Endocrinol Metab 2011;96:1336-43.

38 Partridge A, Gelber S, Gelber RD, et al. Age of menopause among women who remain premenopausal following treatment for early breast cancer: long-term results from international breast cancer Study Group trials V and VI. Eur J Cancer 2007;43:1646-53.

39 Minisini AM, Menis J, Valent F, et al. Determinants of recovery from amenorrhea in premenopausal breast cancer patients receiving adjuvant chemotherapy in the taxane era. Anticancer Drugs 2009;20:503-7.

40 Nelson SM, Yates RW, Fleming R. Serum anti-Mullerian hormone and FSH: prediction of live birth and extremes of response in stimulated cycles implications for individualization of therapy. Human Reproduction 2007;22:2414-21.

41 Hamy A-S, Porcher R, Cuvier C, et al. Ovarian reserve in breast cancer: assessment with anti-Müllerian hormone. Reprod Biomed Online 2014;29:573-80.

42 Leonard RCF, Adamson DJA, Bertelli G, et al. Gnrh agonist for protection against ovarian toxicity during chemotherapy for early breast cancer: the Anglo Celtic group option trial. Ann Oncol 2017;28:1811-6.

43 Pistilli B, Mazouni C, Zingarello A, et al. Individualized prediction of menses recovery after chemotherapy for early-stage breast cancer: a nomogram developed from UNICANCER PACS04 and PACS05 trials. Clin Breast Cancer 2019;19:63-70. 
44 Ford JH, MacCormac L. Pregnancy and lifestyle study: the long-term use of the contraceptive pill and the risk of age-related miscarriage. Hum Reprod 1995;10:1397-402.

45 Baio G, Dawid AP. Probabilistic sensitivity analysis in health economics, research report No. 292. London: Department of Statistical Science, University College London, 2011.

46 Kreke JE, Schaefer AJ, Roberts MS. Simulation and critical care modeling. Curr Opin Crit Care 2004;10:395-8.

47 Richter A, Mauskopf JA. Mm1 Monte Carlo simulation in health care models. Value in Health 1998:1:84-5.

48 Sonnenberg FA, Beck JR. Markov models in medical decision making: a practical guide. Med Decis Making 1993;13:322-38.
49 Richter A, Hauber B, Simpson K, et al. A Monte Carlo simulation for modelling outcomes of AIDS treatment regimens. Pharmacoeconomics 2002;20:215-24.

50 Morse JM. The significance of saturation. Thousand Oaks: Sage Publications, 1995: 147-9.

51 Ajzen I. The theory of planned behavior. Organ Behav Hum Decis Process 1991:50:179-211.

52 Miles MB, Huberman AM. Qualitative data analysis: an expanded sourcebook. Sage, 1994.

53 Tong A, Sainsbury P, Craig J. Consolidated criteria for reporting qualitative research (COREQ): a 32-item checklist for interviews and focus groups. Int J Qual Health Care 2007:19:349-57.

54 Mays N, Pope C. Qualitative research: rigour and qualitative research. BMJ 1995;311:109-12. 